\title{
Gestión del agua marina desalinizada en parcela: adaptación de la fertirrigación y uso conjunto con otras fuentes de agua
}

\author{
V. Martínez-Alvarez ${ }^{1}$, J. F. Maestre-Valero ${ }^{1}$, B. Martin-Gorriz ${ }^{1}$, B. Gallego Elvira1 \\ 1 Escuela Técnica Superior de Ingeniería Agronómica, Universidad Politécnica de Cartagena. Paseo \\ Alfonso XIII, 48. 30203 Cartagena, España; victoriano.martinez@upct.es
}

\begin{abstract}
Resumen: El riego agrícola con Agua Marina Desalinizada (AMD) se ha desarrollado en la última década en el sureste español e Israel. El AMD. Desde el punto de vista agronómico, el AMD se caracteriza por su escasa mineralización y el desequilibrio en su composición, ya que tiene alta concentración de $\mathrm{Na}^{+}, \mathrm{Cl}^{-}$y B ${ }^{3+}$, y baja concentración en $\mathrm{Ca}^{2+}, \mathrm{Mg}^{2+}$ y SO4${ }^{2-}$. Esta singularidad, si no es considerada en el manejo del riego y la fertirrigación, puede llevar a pérdidas en la producción y la calidad de la cosecha. Para evitar estos problemas hay que hacer una adecuada gestión del AMD en parcela, especialmente orientada a adecuar su composición a la necesidad de nutrientes de los cultivos, siendo necesario adaptar los programas de fertirrigación y utilizar posibles mezclas con otras fuentes de agua que puedan aportar $\mathrm{Ca}^{2+}$, $\mathrm{Mg}^{2+}$ y SO42-. En este trabajo se comparan las necesidades de fertirrigación de varios cultivos característicos del sureste español cultivos, bajo sus condiciones de cultivo en el Campo de Cartagena, considerando tres posibles escenarios de suministro de agua: $100 \%$ del riego con agua del trasvase Tajo-Segura, 100\% del riego con AMD, y mezcla al 50\% de ambas fuentes de agua. Los resultados del estudio ponen de manifiesto que la incorporación de AMD al riego hace necesario adaptar los programas de fertirrigación con el fin de compensar su baja mineralización, especialmente en cultivos sin suelo (en sustrato e hidropónico). El coste de esta adaptación es relativamente pequeño en comparación con el incremento de coste del agua de riego. Para algunos cultivos, la suma de ambos incrementos puede hacer peligrar su viabilidad económica, ya que incrementa notablemente los costes anuales de operación.
\end{abstract}

Palabras clave: riego, calidad del agua, fertilización, cotes de producción, sureste español.

\section{Introducción}

La incorporación de agua marina desalinizada (AMD) al sureste español es la principal estrategia recogida en la planificación hídrica española con el fin de hacer frente al déficit estructural de agua, que afecta principalmente al regadío. La revisión de las principales experiencias de riego agrícola con agua desalinizada pone de manifiesto que en numerosos países con clima árido o semiárido, y que además disponen de una agricultura altamente tecnificada, la desalinización de aguas salobres representa una fuente de agua suplementaria desde hace varias décadas. Sin embargo, el riego agrícola con suministros basados en AMD solo se encuentra documentado en España e Israel, y en la última década [1].

En general, la principal ventaja del AMD es su condición de recurso hídrico inagotable y no sujeto a variaciones climáticas, por lo que estratégicamente resulta idóneo para aumentar la disponibilidad de recursos hídricos para riego agrícola en zonas deficitarias. Como principal inconveniente persiste el elevado consumo energético asociado a su producción, generando un 


\section{CONGRESO IBÉRICO DE AGROINGENIERÍA \\ X CONGRESSO IBÉRICO DE AGROENGENHARIA \\ 3 - 6 septiembre 2019, Huesca - España}

coste del agua producto elevado y un nivel de emisiones de gases de efecto invernadero que dificulta las políticas demandadas para el control del cambio climático.

La osmosis inversa se ha generalizado como la tecnología de referencia para la desalinización de agua marina. El AMD resultante presenta una conductividad de 350-600 $\mu \mathrm{Scm}^{-1}$, rango muy adecuado para el riego agrícola. Sin embargo, desde el punto de vista agronómico, el AMD también se caracteriza por su escasa mineralización y el desequilibrio en su composición, ya que tiene alta concentración de $\mathrm{Na}^{+} \mathrm{y} \mathrm{Cl}-\mathrm{y}$ baja concentración en $\mathrm{Ca}^{2+}, \mathrm{Mg}^{2+} \mathrm{y}$ $\mathrm{SO}^{2-}$. Esta singularidad, si no es considerada en el manejo del riego y la fertirrigación, puede llevar a pérdidas significativas en producción y calidad, como ponen de manifiesto varios estudios en cultivos regados únicamente con aguas desalinizadas en Israel [2, 3, 4]. En estas experiencias se han detectado problemas agronómicos que pueden afectar a la productividad de los cultivos, a los costes de fertirrigación y a la conservación de los suelos agrícolas, aspectos del riego con AMD que deben investigarse y analizarse para garantizar su viabilidad económica en el corto plazo y su sostenibilidad ambiental en el medio-largo plazo.

Una de las principales inquietudes en relación al uso del AMD en riego agrícola es su baja concentración de nutrientes esencial como $\mathrm{Ca}^{2+}, \mathrm{Mg}^{2+}$ y SO${ }_{4}^{2-}$. Las aguas naturales y los suelos del sureste español generalmente proporcionan cantidades suficientes de estos nutrientes como para desestimar la necesidad de incorporarlos en los programas de fertilización. Sin embargo, como se pone de manifiesto en la Tabla 1, la concentración de $\mathrm{Ca}^{2+}, \mathrm{Mg}^{2+} \mathrm{y} \mathrm{SO}_{4^{2-}}$ en el AMD es marcadamente inferior, ya que el proceso de osmosis inversa no solo separa las sales perjudiciales, sino también aquellas necesarias para el desarrollo de los cultivos. Por lo tanto, si queremos evitar carencias nutricionales que afecten a la producción, resulta necesario adaptar los programas de fertirrigación para proporcionar las cantidades requeridas de estos nutrientes, con el consiguiente incremento de coste en fertilizantes. En este sentido, Ben-Gal et al. [3] indicaron que para un cultivo de pimiento en invernadero en Israel se producía un incremento del coste de fertilización cuando se empleaba AMD para el riego de $0,50 \$ / \mathrm{m}^{3}$ por los motivos expuestos, lo que representa un preocupante incremento de $3500 \$ /$ ha de cultivo.

Tabla 1. Composición química del AMD de las 4 grandes planta desaladoras que actualmente suministran a la agricultura de la cuenca del Segura. Se incluyen también los valores del trasvase Tajo-Segura y del agua subterránea en el Campo de Cartagena.

\begin{tabular}{|c|c|c|c|c|c|c|}
\hline \multirow{2}{*}{ Parámetro } & \multicolumn{4}{|c|}{ Plantas desaladoras } & \multirow{2}{*}{$\begin{array}{c}\text { Agua del } \\
\text { Acueducto } \\
\text { Tajo-Segura }\end{array}$} & \multirow{2}{*}{$\begin{array}{c}\text { Agua } \\
\text { Subterránea }\end{array}$} \\
\hline & Torrevieja & Águilas & Escombreras & Valdelentisco & & \\
\hline $\begin{array}{c}\text { EC }(\mathrm{dS} \\
\left.\mathrm{m}^{-1}\right)\end{array}$ & 0.46 & $0.48 \pm 0.08$ & 0.54 & $0.54 \pm 0.15$ & $0.85 \pm 0.05$ & $4.51 \pm 0.98$ \\
\hline $\mathrm{Ca}^{2+}\left(\mathrm{mg} \mathrm{L}^{-1}\right)$ & 29 & $14.9 \pm 2.4$ & 20 & $15.6 \pm 1.8$ & $94.6 \pm 4.0$ & $229.5 \pm 39.3$ \\
\hline $\begin{array}{c}\mathrm{Mg}^{2+}(\mathrm{mg} \\
\left.\mathrm{L}^{-1}\right)\end{array}$ & 4.3 & $1.4 \pm 0.5$ & 2.4 & $2.1 \pm 1.1$ & $41.7 \pm 1.1$ & $99 \pm 17$ \\
\hline $\begin{array}{c}\mathrm{SO}_{4}{ }^{2-}(\mathrm{mg} \\
\left.\mathrm{L}^{-1}\right)\end{array}$ & 6.6 & - & 4.0 & - & - & $980 \pm 364$ \\
\hline $\mathrm{Cl}^{-}\left(\mathrm{mg} \mathrm{L}^{-1}\right)$ & 147 & $140 \pm 30$ & 140 & $182 \pm 47$ & $57 \pm 23$ & $972 \pm 608$ \\
\hline $\mathrm{Na}^{+}\left(\mathrm{mg} \mathrm{L}^{-1}\right)$ & 86 & $76 \pm 14$ & 88 & $115 \pm 18$ & $51 \pm 9$ & $573 \pm 153$ \\
\hline $\mathrm{B}^{3+}\left(\mathrm{mg} \mathrm{L}^{-1}\right)$ & 0.56 & $0 . .85 \pm 0.16$ & 0.9 & $0.92 \pm 0.14$ & $0.13 \pm 0.06$ & $1.36 \pm 0.54$ \\
\hline
\end{tabular}

Fuente: [5] 


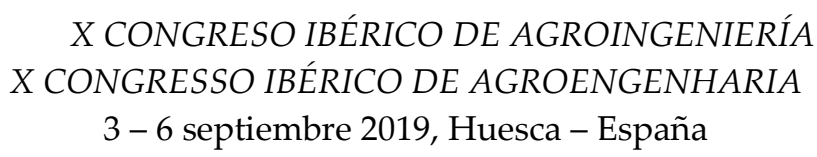

\section{Objetivos}

El objetivo de este trabajo es determinar, de forma analítica, el efecto de la sustitución de las aguas de riego tradicionales por AMD en el coste de fertilización de los cultivos. Para ello se determinarán y comparan las necesidades de fertirrigación de tres cultivos bajo distintos sistemas productivos característicos del sureste español (lechuga Little Gem en sistema hidropónico NFT (Nutrient Film Technique); lechuga Little Gem en suelo; limonero fino en marco tradicional; pimiento dulce en invernadero en sistema hidropónico; y pimiento dulce en invernadero en suelo). Se considerarán tres posibles escenarios de suministro de agua: $100 \%$ del riego con agua del trasvase Tajo-Segura, 100\% del riego con AMD, y mezcla al 50\% de ambas fuentes de agua. La última opción se considera dado que la mezcla del AMD con otras fuentes es la alternativa de gestión más recomendada [6].

El estudio se enmarca en la comarca agrícola del Campo de Cartagena (sureste de España) por ser la región donde se está produciendo un mayor aporte de AMD para riego [5]. Por este motivo, todos los condicionantes del estudio (composición química de las aguas, prácticas de cultivo, costes de cultivo, producciones, etc.) son los característicos de esta zona.

El análisis de los escenarios de cultivo y suministro planteados, junto con los costes de fertilizantes propios de la zona de estudio, aportará información suficientemente representativa de las necesidades de adaptación de la fertirrigación cuando se emplea AMD para riego, así como de su impacto económico bajo condiciones de cultivo características del sureste español.

\section{Materiales y métodos}

\subsection{Datos de cultivo y necesidades de fertilización}

Uno de los condicionantes para la selección de cultivos y sistemas de producción ha sido disponer de suficiente información secundaria sobre los mismos para poder analizar el impacto económico de la adaptación del programa de fertirrigación a los distintos escenarios de suministro de agua de riego considerados.

Los datos económicos que caracterizan a los cultivos de lechuga Little Gem en sistema hidropónico NFT y en suelo se han obtenido de un trabajo previo de los autores [7]. Las recomendaciones de fertilización en suelo $\left(\mathrm{N}-\mathrm{P}_{2} \mathrm{O}_{5}-\mathrm{K}_{2} \mathrm{O}-\mathrm{CaO}-\mathrm{MgO}\right.$ en $\mathrm{kg} / \mathrm{ha}$; 60-50-116-19-10) han sido tomadas de Rincón [8] mientras que la solución nutritiva ideal para el caso de hidropónico NFT ha sido proporcionada por agricultores con experiencia en este tipo de cultivo $\left(\mathrm{NO}^{3-}-\mathrm{H}_{2} \mathrm{PO}_{4}^{-}-\mathrm{SO}_{4}^{-2}-\mathrm{HCO}_{3}^{-}-\mathrm{NH}_{4}^{+}-\mathrm{K}^{+}-\mathrm{Ca}^{+2}-\mathrm{Mg}^{+2}\right.$ en mmol/L; $11,0-1,0-2,5-0,5-1-7,4-$ $4,4-1,2)$.

En el caso de limonero fino en marco tradicional ( $7 \times 6=238$ árboles/ha), tanto la caracterización económica del cultivo como las recomendaciones de fertilización se han obtenido de [9].

Finalmente, la solución nutritiva ideal para el cultivo del pimiento dulce en invernadero con sistema hidropónico $\left(\mathrm{NO}_{3}-\mathrm{H}_{2} \mathrm{PO}^{4-}-\mathrm{SO}_{4}^{-2}-\mathrm{HCO}_{3}-\mathrm{NH}_{4}^{+}-\mathrm{K}^{+}-\mathrm{Ca}^{+2}-\mathrm{Mg}^{+2}\right.$ en mmol/L; 14 - 1,7 $2,0-0,5-0,5-5,9-5,0-2,5)$ o sobre suelo $\left(\mathrm{N}-\mathrm{P}_{2} \mathrm{O}_{5}-\mathrm{K}_{2} \mathrm{O}-\mathrm{CaO}-\mathrm{MgO}\right.$ en $\mathrm{kg} / \mathrm{ha}$; 280-100-460-110-170) se han obtenido de Reche [10], mientras que los datos económicos son los proporcionados por ECREA [11].

\subsection{Caracterización química de los suministros de agua de riego}

Se dispone de dos fuentes de suministro de agua de riego. La primera es la proporcionada por la Comunidad de Regantes del Campo de Cartagena, cuyo origen proviene mayoritariamente del trasvase Tajo-Segura. La segunda es la proporcionada por la planta desaladora de Escombreras, cuya producción actual $(\approx 21 \mathrm{hm} 3 /$ año $)$ se dedica prácticamente en su totalidad al riego de cultivos en el Campo de Cartagena. La composición química característica 


\section{CONGRESO IBÉRICO DE AGROINGENIERÍA \\ X CONGRESSO IBÉRICO DE AGROENGENHARIA \\ 3 - 6 septiembre 2019, Huesca - España}

de estas fuentes se ha obtenido mediante análisis agronómicos de calidad de agua de riego y es la recogida en la Tabla 1. La calidad de agua considerada para el escenario de mezcla al 50\% de AMD y agua del trasvase Tajo-Segura es la correspondiente a los valores medios de cada uno de los componentes químicos considerados. El precio del agua suministrada por la Comunidad de Regantes del Campo de Cartagena es de 0,15€, mientras que el correspondiente al AMD es de $0,50 €$

\subsection{Precio de los fertilizantes}

Los precios de los fertilizantes considerados en el análisis son los valores medios proporcionados por tres empresas distribuidoras de agroquímicos en la zona de estudio para el año 2018, y se recogen en la Tabla 2.

Tabla 2. Precio de los fertilizantes considerados en el estudio

\begin{tabular}{|c|c|c|}
\hline \multicolumn{2}{|c|}{ Fertilizante } & \multirow{2}{*}{$\begin{array}{c}\text { Precio }(€ / \mathbf{k g}) \\
0,644\end{array}$} \\
\hline Ácido Fosfórico & $\mathrm{H}_{3} \mathrm{PO}_{4}$ & \\
\hline Ácido Nítrico & $\mathrm{HNO}_{3}$ & 0,442 \\
\hline Nitrato Potásico & $\mathrm{KNO}_{3}$ & 1,075 \\
\hline Nitrato Amónico & $\mathrm{NH}_{4} \mathrm{NO}_{3}$ & 0,357 \\
\hline Sulfato Amónico & $\left(\mathrm{NH}_{4}\right)_{2} \mathrm{SO}_{4}$ & 0,252 \\
\hline Sulfato Potásico & $\mathrm{K}_{2} \mathrm{SO}_{4}$ & 0,714 \\
\hline Sulfato Magnésico & $\mathrm{MgSO}_{4}$ & 0,690 \\
\hline Fosfato monoamónico & $\mathrm{NH}_{4} \mathrm{H}_{2} \mathrm{PO}_{4}$ & 1,029 \\
\hline Fosfato monopotásico & $\mathrm{KH}_{2} \mathrm{PO}_{4}$ & 1,457 \\
\hline Nitrato Magnésico & $\mathrm{Mg}\left(\mathrm{NO}_{3}\right)_{2}$ & 0,617 \\
\hline Nitrato Cálcico & $\mathrm{Ca}\left(\mathrm{NO}_{3}\right)_{2}$ & 0,440 \\
\hline Complejos mezcla de microelementos & $\mathrm{Fe}, \mathrm{Zn}, \mathrm{Cu}, \mathrm{B}, \mathrm{Mn}$ y Mo & 8,235 \\
\hline
\end{tabular}

\subsection{Ajuste de los programas de fertirrigación}

La metodología aplicada para el ajuste de los programas de fertirrigación en cada escenario de riego ha sido la habitual en el cálculo de soluciones nutritivas en cultivos en suelo y sin suelo [12]. Por lo general, en cultivos sin suelo, se ha seguido el procedimiento de cálculo descrito por Sonnenveld [13].

Una vez ajustados los programas de fertirrigación, se ha determinado la dosificación de fertilizantes. Finalmente, a partir de las dosificaciones consideradas y del volumen anual de riego se obtienen las cantidades a aportar de cada fertilizante.

Las soluciones adoptadas han sido contrastadas con un panel de agricultores especializados en cada cultivo que, en base a su experiencia, han confirmado la representatividad de las mismas en cada escenario de suministro de agua de riego.

\section{Resultados y discusión}

\subsection{Lechuga Little Gem en sistema hidropónico NFT}

Para el estudio de este sistema productivo se ha considerado el análisis de una campaña completa, en la que se producen 9 ciclos de cultivo al año, con una densidad de plantación de 165.000 lechugas/ha. El consumo hídrico es de $750 \mathrm{~m}^{3} / \mathrm{ha}$-ciclo, representando un total de 6750 $\mathrm{m}^{3} /$ ha-año. La Tabla 3 sintetiza el consumo de fertilizantes para cada escenario, así como el coste asociado de los mismos. También se incluye el incremento del coste de fertilización en cada escenario, así como el incremento de coste por fertilización y uso de AMD. 


\section{CONGRESO IBÉRICO DE AGROINGENIERÍA \\ X CONGRESSO IBÉRICO DE AGROENGENHARIA \\ 3 - 6 septiembre 2019, Huesca - España}

Teniendo en cuenta que los costes de operación anuales del cultivo de lechuga Little Gem en sistema hidropónico NFT ascienden a $96.607 € /$ ha (Maestre-Valero et al., 2018), el incremento de costes de fertilización al reemplazar el $100 \%$ de agua de riego con AMD únicamente representa un $1,6 \%$ de los mismos, mientras que el incremento de coste por fertilización y agua de riego asciende al 4,1\%. En el caso de reemplazar solamente el $50 \%$, estos incrementos serían del $0,3 \%$ y $1,6 \%$ respectivamente.

Tabla 3. Cantidades de fertilizantes, agua de riego aplicada y costes para lechuga Little Gem en sistema hidropónico NFT

\begin{tabular}{cccc}
\hline Fertilizante (kg/año) & E1: 0\% AMD & E2: 50\% AMD & E3: 100\% AMD \\
\hline Ácido Fosfórico & 945,0 & 945,0 & 0,0 \\
Ácido Nítrico & 1401,7 & 551,2 & 488,2 \\
Nitrato Potásico & 2784,2 & 2286,1 & 1774,3 \\
Nitrato Amónico & 534,6 & 534,6 & 0 \\
Sulfato Amónico & 0 & 0 & 0 \\
Sulfato Potásico & 1905,9 & 2329,5 & 2764,8 \\
Sulfato Magnésico & 0 & 515,3 & 1845,4 \\
Fosfato monoamónico & 0 & 0 & 776,2 \\
Fosfato monopotásico & 0 & 0 & 0,0 \\
Nitrato Magnésico & 0 & 0 & 0,0 \\
Nitrato Cálcico & 3003,4 & 4330,2 & 5671,6 \\
Microelementos & 168,7 & 168,7 & 168,7 \\
\hline Coste Fertilización ( $(€ / \mathbf{a n ̃ o})$ & 8481,7 & 8812,3 & 10052,5 \\
$\Delta$ Coste Fert. (€/año) & - & 330,6 & 1570,7 \\
Coste Fert + Agua (€/año) & 9494,2 & 11006,1 & 13423,5 \\
$\Delta$ Coste Fert. + Agua (€/año) & - & 1511,9 & 3929,3 \\
\hline
\end{tabular}

\subsection{Lechuga Little Gem en suelo}

Para este caso se ha considerado el manejo habitual en el Campo de Cartagena, que consiste en producir dos ciclos de lechuga de otoño-invierno en cada campaña. La densidad de plantación es de 155.000 lechugas/ha. El consumo hídrico es de $1850 \mathrm{~m} 3 / \mathrm{ha} \cdot \mathrm{ciclo}$, representando un total de $3700 \mathrm{~m}^{3} / \mathrm{ha} \cdot a n ̃ o$. La Tabla 4 sintetiza los resultados para cada escenario de riego considerado.

Teniendo en cuenta que los costes de operación anuales del cultivo de lechuga Little Gem en suelo ascienden a $18.360 € /$ ha [7], el incremento de costes de fertilización al reemplazar el 100\% de agua de riego con AMD sólo representa un 1,0\% de los mismos, mientras que el incremento de coste por fertilización y agua de riego asciende al $8,1 \%$. En el caso de reemplazar solamente el $50 \%$, estos incrementos serían del $0,0 \%$ y 3,5\% respectivamente.

\subsection{Limonero Fino en marco tradicional}

Se ha considerado una plantación de limonero "fino 95" (Citrus limon L. Burm F.) sobre sobre Citrus macrophylla, con un marco de plantación tradicional de $7 \times 6 \mathrm{~m}$, lo que supone 238 árboles/ha. La producción característica de este sistema de cultivo en la zona de estudio es de $45.000 \mathrm{~kg} / \mathrm{ha}$, mientras que el consumo de agua de riego es de $6000 \mathrm{~m}^{3} / \mathrm{ha}$-año. La Tabla 5 sintetiza los resultados para cada escenario considerado.

Los costes de operación anuales del cultivo de limón asciende a 5.551 €/ha [14], por lo que el incremento de costes de fertilización en el escenario 3 (100\% AMD) representa un 1,0\% de los mismos, mientras que el incremento de coste por fertilización y agua de riego asciende al 38,8\%. En el caso de reemplazar solamente el 50\%, los incrementos serían del 0,0\% y 18,9\% respectivamente. 


\section{CONGRESO IBÉRICO DE AGROINGENIERÍA \\ X CONGRESSO IBÉRICO DE AGROENGENHARIA \\ 3 - 6 septiembre 2019, Huesca - España}

Tabla 4. Cantidades de fertilizantes, agua de riego aplicada y costes para lechuga Little Gem en suelo

\begin{tabular}{cccc}
\hline Fertilizante (kg/año) & E1: 0\% AMD & E2: $\mathbf{5 0} \%$ AMD & E3: 100\% AMD \\
\hline Ácido Fosfórico & 0 & 0 & 0 \\
Ácido Nítrico & 0 & 0 & 0 \\
Nitrato Potásico & 336,6 & 336,6 & 321,7 \\
Nitrato Amónico & 213,1 & 216,0 & 155,1 \\
Sulfato Amónico & 0 & 0 & 0 \\
Sulfato Potásico & 0 & 0 & 0 \\
Sulfato Magnésico & 0 & 0 & 227,8 \\
Fosfato monoamónico & 0 & 0 & 0 \\
Fosfato monopotásico & 191,3 & 191,3 & 191,3 \\
Nitrato Magnésico & 0 & 0 & 0 \\
Nitrato Cálcico & 0 & 0 & 151,8 \\
Microelementos & 37,0 & 37,0 & 37,0 \\
\hline Coste Fertilización $(\boldsymbol{€} / \mathbf{a n ̃ o})$ & 1021,3 & 1022,4 & 1208,5 \\
$\Delta$ Coste Fert. $(\boldsymbol{(} / \mathbf{a n ̃ o})$ & - & 1,1 & 187,2 \\
Coste Fert + Agua $(\boldsymbol{€} / \mathbf{a n ̃ o})$ & 1576,3 & 2224,9 & 3058,5 \\
$\Delta$ Coste Fert. + Agua $(\boldsymbol{(} / \mathbf{a n ̃ o})$ & - & 648,6 & 1482,2 \\
\hline
\end{tabular}

Tabla 5. Cantidades de fertilizantes, agua de riego aplicada y costes para Limonero Fino en marco tradicional.

\begin{tabular}{cccc}
\hline Fertilizante (kg/año) & E1: 0\% AMD & E2: 50\% AMD & E3: 100\% AMD \\
\hline Ácido Fosfórico & 122,6 & 122,6 & 122,6 \\
Ácido Nítrico & 0 & 0 & 0 \\
Nitrato Potásico & 242,6 & 242,6 & 233,5 \\
Nitrato Amónico & 444,4 & 444,4 & 427,2 \\
Sulfato Amónico & 0 & 0 & 0 \\
Sulfato Potásico & 0 & 0 & 0 \\
Sulfato Magnésico & 0 & 0 & 59,1 \\
Fosfato monoamónico & 0 & 0 & 0 \\
Fosfato monopotásico & 0 & 0 & 0 \\
Nitrato Magnésico & 0 & 0 & 0 \\
Nitrato Cálcico & 0 & 0 & 63,5 \\
Microelementos & 17,0 & 17,0 & 16,9 \\
\hline Coste Fertilización $(\boldsymbol{(} / \mathbf{a n ̃ o})$ & 638,0 & 638,0 & 690,8 \\
$\Delta$ Coste Fert. $(\boldsymbol{(} / \mathbf{a n ̃ o})$ & - & 0 & 52,8 \\
Coste Fert + Agua (€/año) & 1538,0 & 2588,0 & 3690,8 \\
$\Delta$ Coste Fert. + Agua (€/año) & - & 1050,0 & 2152,8 \\
\hline
\end{tabular}

\subsection{Pimiento dulce en invernadero con sistema hidropónico}

Las técnicas de producción de pimiento en invernadero difieren muchos entre regiones. Así, en Almería, donde existen unas 8.000 ha de invernadero de pimiento, se realizan cultivos de otoño (Agosto-Febrero). Sin embargo, en el campo de Cartagena, donde se cultivan cerca de 1400 ha de pimiento, se realizan cultivos de primavera (Noviembre-Julio). Para este último caso, la densidad de plantación habitual es de 25.000 plantas/ha, dando lugar a producciones características en torno a los $80.000 \mathrm{~kg} / \mathrm{ha}$, con consumos de agua de riego cercanos a los 9000 $\mathrm{m}^{3} /$ ha. Los resultados obtenidos bajo este escenario se sintetizan en la Tabla 6 . 


\section{CONGRESO IBÉRICO DE AGROINGENIERÍA \\ X CONGRESSO IBÉRICO DE AGROENGENHARIA \\ 3 - 6 septiembre 2019, Huesca - España}

Los costes de operación anuales del cultivo de para pimiento en sustrato de coco en el Campo de Cartagena asciende a $74.244 € /$ ha [14], por lo que el incremento de costes de fertilización en el escenario 3 (100\% AMD) representa un 4,7\% de los mismos, mientras que el incremento de coste por fertilización y agua de riego asciende al 9,0\%. En el caso de reemplazar solamente el 50\% del suministro por AMD, los incrementos serían del 2,0\% en el coste de fertilizantes y del 4,1\% en el coste por fertilización y agua de riego.

Tabla 6. Cantidades de fertilizantes, agua de riego aplicada y costes para pimiento dulce con sistema hidropónico

\begin{tabular}{cccc}
\hline Fertilizante (kg/año) & E1: 0\% AMD & E2: 50\% AMD & E3: 100\% AMD \\
\hline Ácido Fosfórico & 2142,0 & 2142,0 & 781,2 \\
Ácido Nítrico & 1134,0 & 0 & 0 \\
Nitrato Potásico & 4513,1 & 4467,6 & 3475,8 \\
Nitrato Amónico & 352,8 & 352,8 & 352,8 \\
Sulfato Amónico & 0 & 0 & 0 \\
Sulfato Potásico & 0 & 0 & 0 \\
Sulfato Magnésico & 0 & 2527,0 & 5098,4 \\
Fosfato monoamónico & 0 & 0 & 0 \\
Fosfato monopotásico & 0 & 0 & 1322,8 \\
Nitrato Magnésico & 1868,4 & 1084,1 & 253,7 \\
Nitrato Cálcico & 5171,0 & 6940,0 & 8728,5 \\
Microelementos & 225,0 & 225,0 & 225,0 \\
\hline Coste Fertilización (€/año) & $12.136,4$ & $13.625,4$ & $15.659,6$ \\
$\Delta$ Coste Fert. (€/año) & - & 1489,0 & 3523,2 \\
Coste Fert + Agua (€/año) & $13.486,4$ & $16.550,4$ & $20.159,6$ \\
$\Delta$ Coste Fert. + Agua (€/año) & - & 3064,0 & 6673,2 \\
\hline
\end{tabular}

\subsection{Pimiento dulce en invernadero sobre suelo}

Se trata de un sistema productivo similar al anterior, pero que se desarrolla directamente sobre suelo en lugar de sustrato. Las producciones suelen ser ligeramente inferiores para similares densidades de cultivo y dosis de riego. Los resultados obtenidos bajo este escenario se sintetizan en la Tabla 7.

Tabla 7. Cantidades de fertilizantes, agua de riego aplicada y costes para pimiento dulce sobre suelo

\begin{tabular}{cccc}
\hline Fertilizante (kg/año) & E1: 0\% AMD & E2: 50\% AMD & E3: 100\% AMD \\
\hline Ácido Fosfórico & 0 & 0 & 0 \\
Ácido Nítrico & 0,0 & 0 & 0 \\
Nitrato Potásico & 773,4 & 764,3 & 755,2 \\
Nitrato Amónico & 324,0 & 180,0 & 25,2 \\
Sulfato Amónico & 0 & 0 & 0 \\
Sulfato Potásico & 0 & 0 & 0 \\
Sulfato Magnésico & 0 & 0 & 0 \\
Fosfato monoamónico & 0 & 0 & 0 \\
Fosfato monopotásico & 189,8 & 189,8 & 189,8 \\
Nitrato Magnésico & 230,6 & 461,3 & 692,0 \\
Nitrato Cálcico & 233,2 & 408,2 & 622,0 \\
Microelementos & 90,0 & 90,0 & 90,0 \\
\hline Coste Fertilización ( $(€ /$ año) & 2209,5 & 2367,5 & 2538,9 \\
$\Delta$ Coste Fert. $(\boldsymbol{€} / \mathbf{a n ̃ o})$ & - & 158,0 & 329,4 \\
Coste Fert + Agua $(\boldsymbol{(} / \mathbf{a n ̃ o})$ & 3559,5 & 5292,5 & 7038,9 \\
$\Delta$ Coste Fert. + Agua $(\boldsymbol{\epsilon} / \mathbf{a n ̃ o})$ & - & 1733,1 & 3479,4 \\
\hline
\end{tabular}




\section{CONGRESO IBÉRICO DE AGROINGENIERÍA \\ X CONGRESSO IBÉRICO DE AGROENGENHARIA \\ 3 - 6 septiembre 2019, Huesca - España}

Según el ECREA [11] los costes de operación anuales (costes directos + mano de obra + costes indirectos) del pimiento dulce en invernadero sobre suelo asciende a $30.799 € / \mathrm{ha}$. Por tanto, el incremento de costes de fertilización en el escenario 3 (100\% AMD) representa un 1,1\% de los costes anuales de operación, mientras que el incremento de coste por fertilización y agua de riego asciende al 11,3\%. En el caso de reemplazar solamente el 50\% del suministro por AMD, los incrementos serían del $0,5 \%$ en el coste de fertilizantes y del 5,6\% en el coste por fertilización y agua de riego.

\section{Discusión y Conclusiones}

Tanto el agua de riego proveniente del trasvase Tajo-Segura y como de la planta desalinizadora carece de contenidos significativos de N, P y K, por lo que las necesidades de estos nutrientes tienen que ser suministradas con independencia del origen del agua. Sin embargo, el agua del trasvase Tajo-Segura aporta importantes dosis $\mathrm{de} \mathrm{Ca}^{2+} \mathrm{y} \mathrm{Mg}^{2+}$, que suelen ser suficiente en el caso de cultivos en suelo, y que reducen considerablemente el aporte de los mismos en el caso de los cultivos hidropónicos. Además, el micronutriente boro es abundante en el AMD, por lo que debería suprimirse en los programas de fertirrigación para evitar problemas de fitotoxicidad en cultivos sensibles.

La Figura 1 muestra que el incremento de los costes de fertilización al reemplazar el 100\% de agua de riego con AMD es significativo en los cultivos hidropónicos (1500-3500 €/ha-año) y muy reducido en los cultivos sobre suelo (50-330 €/ha·año). Sin embargo, cuando se relativizan estos costes al compararlos con los costes anuales de operación del cultivo, se pone de manifiesto que, con la excepción del cultivo hidropónico de pimiento, el incremento representa sólo entre el 1 y el $2 \%$ de dichos costes. La Figura 1 también muestra que el incremento de los costes de agua al reemplazar el 100\% de agua de riego con AMD se encuentra entre 1000 y $3000 € /$ ha.año. Este incremento puede representar un porcentaje importante de los costes anuales de operación del cultivo, como ocurre en el caso del limonero (38\%).

La Figura 2 muestra que el incremento del coste total al reemplazar el 100\% es más importante en valor absoluto para los cultivos hidropónicos, pero en valor relativo se sitúa entre el 5 y el 10\% para todos los cultivos, con la excepción del limonero, donde alcanza el 39\%.

Finalmente, se puede concluir que la incorporación de AMD al riego hace necesario adaptar los programas de fertirrigación con el fin de compensar su baja mineralización, especialmente en cultivos sin suelo (en sustrato e hidropónico). El coste de esta adaptación es relativamente pequeño en comparación con el incremento de coste del agua de riego. Para algunos cultivos, la suma de ambos incrementos puede hacer peligrar su viabilidad económica, ya que incrementa notablemente los costes anuales de operación.
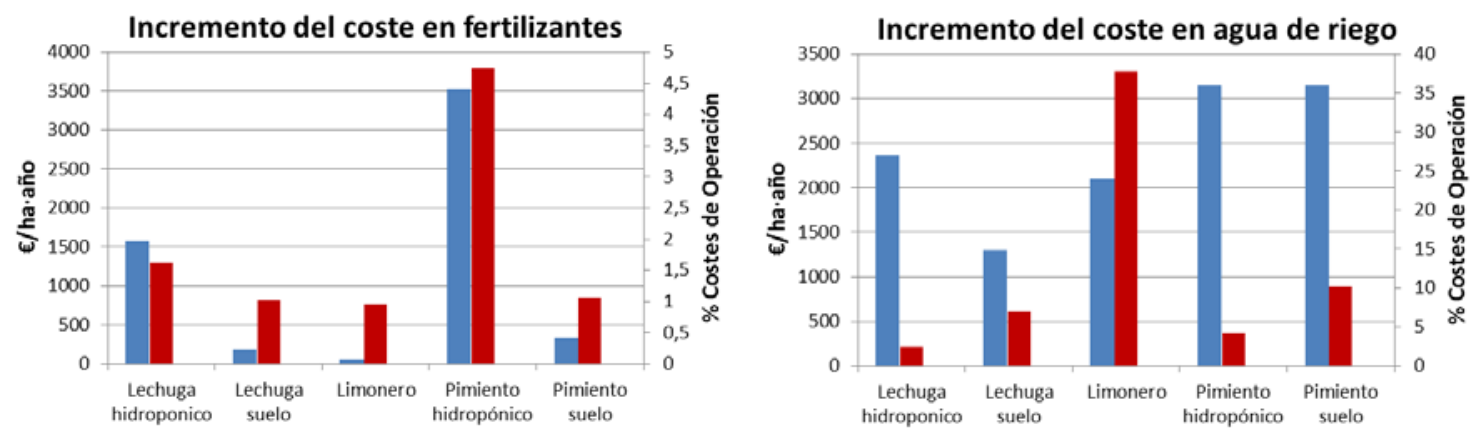

Figura 1. Incrementos de los costes de fertilización (Izda.) y del agua de riego (Dcha.) al reemplazar el $100 \%$ de agua de riego con AMD. 
X CONGRESO IBÉRICO DE AGROINGENIERÍA

X CONGRESSO IBÉRICO DE AGROENGENHARIA

3 - 6 septiembre 2019, Huesca - España

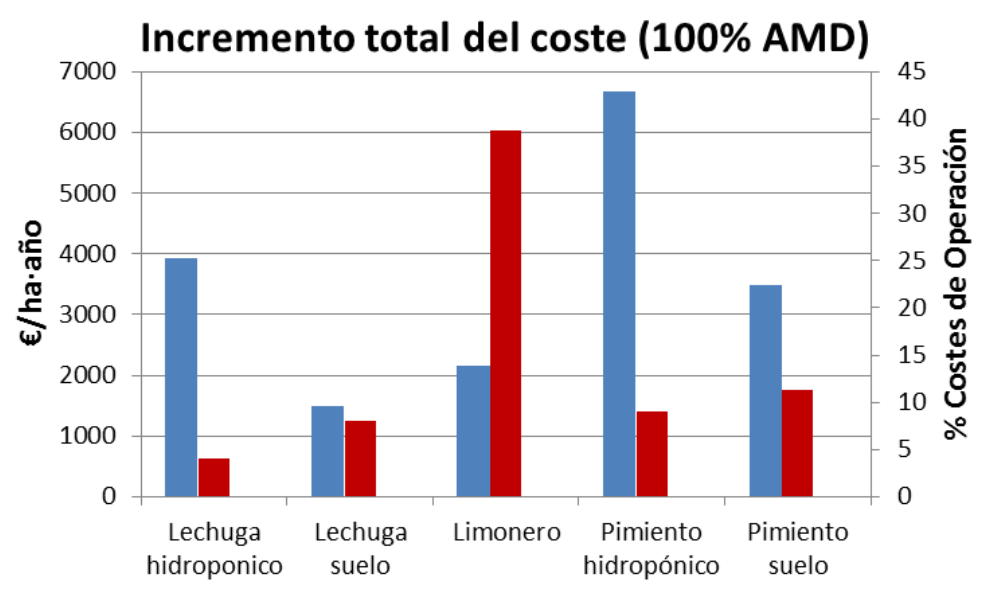

Figura 2. Incremento del coste total de operación al reemplazar el 100\% de agua de riego con AMD.

\section{Agradecimientos}

La realización de este trabajo ha sido posible gracias a (1) la financiación del Ministerio de Economía y Competitividad y el Fondo Europeo de Desarrollo Regional (FEDER) mediante el proyecto RIDESOST (AGL2017-85857-C2-2-R), y (2) la financiación del Proyecto Europeo LIFE DESEACROP (LIFE16-ENV-ES-000341). También se agradece la colaboración de la Catedra Trasvase y Sostenibilidad Jose Manuel Claver Valderas.

\section{Referencias}

1. Martínez-Alvarez V., Martin-Gorriz B., Soto-García M. Seawater desalination for crop irrigation - A review of current experiences and revealed key issues. Desalination. 2016, 381, 58-70.

2. Yermiyahu U., Tal A., Ben-Gal A., Bar-Tal A., Tarchitzky J., Lahav O. Rethinking desalinated water quality and agriculture. Science. 2007, 318, 920-921.

3. Ben-Gal A., Yermiyahu U., Cohen S. Fertilization and blending alternatives for irrigation with desalinated water. J. Environ. Qual. 2009, 38, 529-536.

4. Bar-Tal A., Yermiyahu U., Ben-Gal A., Schwartz A., Faingold I., Seligmann R. Optimization of calcium and magnesium concentrations for fertigation of tomato with desalinated water. Isr. J. Plant Sci. 2017, DOI: 10.1080/07929978.2017.1288466.

5. Martínez-Alvarez V., González-Ortega M.J., Martin-Gorriz B., Soto-García B., Maestre-Valero J.F. The use of desalinated seawater for crop irrigation in the Segura River Basin (south-eastern Spain). Desalination. 2017, 422, 153-164.

6. Avni N., Eben-Chaime M., Oron G. Optimizing desalinated sea water blending with other sources to meet magnesium requirements for potable and irrigation waters. Water Research. 2013, 47, 2164-2176.

7. Maestre-Valero J.F., Martín-Górriz B., Soto García M., Martínez-Mate M.A., Martínez Alvarez V. Producing lettuce in soil-based or in soilless outdoor systems. Which is more economically profitable? Agric Water Manag. 2018, 206, 48-55.

8. Rincón L. Requerimientos de nutrientes en fertirrigación de lechugas especiales y minilechugas. Vida Rural. 2008, 266, $56-60$.

9. García, J. Análisis del sector del limonero y evaluación económica de su cultivo. Instituto Murciano de Investigación y Desarrollo Agrario y Alimentario. Murcia, 2014, 142 pp.

10. Reche J. Cultivo del pimiento Dulce en invernadero. Consejería de Agricultura y Pesca de ANDALUCIA. Sevilla, 2010, 291 pp.

11. ECREA. Estudios de Costes y Rentas de las Explotaciones Agrarias. Resultados técnico-económicos. Cultivos hortícolas Andalucía, Castilla y León, Castilla-La Mancha, Extremadura, Murcia y Comunidad Valenciana. Ministerio de Agricultura, Alimentación y Medio Ambiente. Madrid, 2014, $57 \mathrm{pp}$. 


\section{CONGRESO IBÉRICO DE AGROINGENIERÍA \\ X CONGRESSO IBÉRICO DE AGROENGENHARIA \\ 3 - 6 septiembre 2019, Huesca - España}

12. Santos B., Ríos D. Cálculo de Soluciones Nutritivas En suelo y sin suelo. Servicio de Agricultura y Desarrollo Rural. Cabildo Insular de Tenerife. Tenerife, 2016, 111 pp.

Sonnenveld C., Straver N. Nutrient solutions for vegetables and flowers grown in water or substrates. Voedingsoplossingen glastuinbouw nº 8. Glasshouse Crops Research Station. Naaldwijk, 1994, 45 pp.

13. García, J. Análisis del sector del limonero y evaluación económica de su cultivo. Instituto Murciano de Investigación y Desarrollo Agrario y Alimentario. Murcia, 2014, 142 pp.

14. López-Marín J., Angosto J.L., González A. El cultivo de pimientos en el campo de Cartagena. 2014, https://issuu.com/horticulturaposcosecha/docs/130710josefalopezpimientocartagenao 